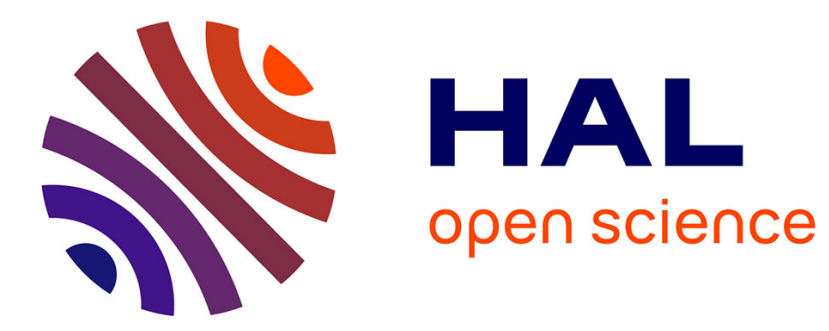

\title{
COEXISTENCE DES FORMES ALLONGÉE ET APLATIE DANS 187Au
}

M. Deleplanque, C. Gerschel, M. Ishihara, N. Perrin, C. Bourgeois, M. Desthuilliers, J. Husson, P. Kilcher, J. Letessier, V. Berg

\section{- To cite this version:}

M. Deleplanque, C. Gerschel, M. Ishihara, N. Perrin, C. Bourgeois, et al.. COEXISTENCE DES FORMES ALLONGÉE ET APLATIE DANS 187Au. Colloquium on Nuclear Surface, 1975, Dijon, France. pp.C5-105-C5-105, 10.1051/jphyscol:1975522 。 jpa-00216377

\section{HAL Id: jpa-00216377 https://hal.science/jpa-00216377}

Submitted on 1 Jan 1975

HAL is a multi-disciplinary open access archive for the deposit and dissemination of scientific research documents, whether they are published or not. The documents may come from teaching and research institutions in France or abroad, or from public or private research centers.
L'archive ouverte pluridisciplinaire HAL, est destinée au dépôt et à la diffusion de documents scientifiques de niveau recherche, publiés ou non, émanant des établissements d'enseignement et de recherche français ou étrangers, des laboratoires publics ou privés. 


\title{
COEXISTENCE DES FORMES ALLONGÉE ET APLATIE DANS ${ }^{187}$ Au
}

\author{
$\left({ }^{1}\right)$ M. A. DELEPLANQUE, C. GERSCHEL, M. ISHIHARA (*), N. PERRIN \\ $\left({ }^{2}\right)$ C. BOURGEOIS, M. G. DESTHUILLIERS $\left({ }^{* *}\right)$, J. P. HUSSON, P. KILCHER, \\ J. LETESSIER et V. BERG \\ Institut de Physique Nucléaire, B.P. no 1, 91406 Orsay, France
}

\begin{abstract}
Résumé. - ${ }^{187} \mathrm{Au}$ a été étudié simultanément par réaction ${ }^{175} \mathrm{Lu}\left({ }^{16} \mathrm{O}, 4 \mathrm{n}\right){ }^{187} \mathrm{Au}$ au cyclotron à énergie variable d'Orsay, et par étude de la désintégration radioactive de ${ }^{187} \mathrm{Hg}\left(T_{1 / 2}=133 \pm 13 \mathrm{~ns}\right)$ à l'aide du séparateur Isocèle en ligne sur le synchrocyclotron de l'IPN d'Orsay. Les résultats obtenus ont été comparés à ceux du cour pair-pair ${ }^{186} \mathrm{Pt}$ formé par réaction ${ }^{174} \mathrm{Yb}\left({ }^{16} \mathrm{O}, 4 \mathrm{n}\right){ }^{186} \mathrm{Pt}$.

On a ainsi mis en évidence deux bandes découplées construites dans les couches $h 11 / 2$ et $\mathrm{h} 9 / 2$ indiquant des déformations respectivement oblate et prolate comme dans ${ }^{189} \mathrm{Au}$ [1]. Dans la bande h $9 / 2$, bien développée, de même que dans le cœur ${ }^{186} \mathrm{Pt}$, on a pu mettre en évidence un effet de backbending : ceci permet de penser qu'il est essentiellement dû à une paire de protons $h 11 / 2$ (blocking du proton $\mathrm{h}$ 9/2 aligné sur le cœur).
\end{abstract}

\begin{abstract}
Au}$ has been investigated simultaneously by means of ${ }^{175} \mathrm{Lu}\left({ }^{16} \mathrm{O}, 4 \mathrm{n}\right){ }^{187} \mathrm{Au}$ reaction using the Orsay heavy-ion cyclotron, and by radioactive decay of ${ }^{187} \mathrm{Hg}\left(T_{1 / 2}=133 \pm 13 \mathrm{~ns}\right)$ obtained with the mass separator Isocele on line with the Orsay synchrocyclotron. The results are compared with those obtained on ${ }^{186} \mathrm{Pt}$ from ${ }^{174} \mathrm{Yb}\left({ }^{16} \mathrm{O}, 4 \mathrm{n}\right){ }^{186} \mathrm{Pt}$ reaction.

Two decoupled bands are built on the $\mathrm{h} 11 / 2$ and $\mathrm{h} 9 / 2$ orbitals which indicate oblate and prolate shapes respectively, as in ${ }^{189} \mathrm{Au}$ [1]. The $\mathrm{h} 9 / 2$ band in ${ }^{187} \mathrm{Au}$ and the yrast band of ${ }^{186} \mathrm{Pt}$ show backbending behaviour : this suggests that a $\mathrm{h} 11 / 2$ proton pair becomes aligned with the rotational spin of the core.
\end{abstract}

\section{Bibliographie}

[1] Berg, V., Deleplanque, M. A., Gerschel, C., Perrin, N., Int. Conf. on Nuclear Structure (Amsterdam) (1974) 113, à paraître.

Berg, V., Höglund, A., Foucher, R., Int. Conf. on Nuclear Structure (Amsterdam) 1974, 113 et Nucl. Phys. (à parấtre).

(') Réactions (IL, $x n$ )

$\left({ }^{2}\right)$ Radioactivité.

(*) Institute of Nuclear Study, Tokyo, Japan.

${ }^{* *}$ ) C.S.N.S.M. Orsay, France. 